\title{
Crop Selection based on Fuzzy TOPSIS using Entropy Weights
}

\author{
A. Sahaya Sudha, PhD \\ Assistant Professor, Department of Mathematics, \\ Nirmala College for women, Coimbatore, \\ TamilNadu, India
}

\author{
J. Rachel Inba Jeba \\ M.Phil., Scholar, Department of Mathematics, \\ Nirmala College for women, Coimbatore, \\ TamilNadu, India
}

\begin{abstract}
The objective of this paper is to extend the TOPSIS to the fuzzy environment. FUZZY TOPSIS is one of the various models of multiple attributes decision making with triangular fuzzy values that so far diverse models have been introduced. The concepts represented in the decision data wherein the crisp value are inadequate to model in real-life situations. In this paper the rating of each alternatives are described by triangular fuzzy numbers, and the weights of each criterion are found by entropy. According to the concept of TOPSIS, a closeness coefficient is defined to determine the raking by calculating the distance of both the fuzzy positive-ideal solution and fuzzy negative-ideal solution. The proposed methods have been applied for five different crops with various criteria for a better and more accurate outputs.
\end{abstract}

\section{Keywords}

TOPSIS, Fuzzy TOPSIS, Triangular Fuzzy Numbers.

\section{INTRODUCTION}

Decision-making problem is the process of finding the best option from all available feasible alternatives. In almost all such problems the multiplicity of criteria for judging the alternatives is pervasive. Technique for order performance by similarity to ideal solution (TOPSIS), one of the known classical MCDM method, was first developed by (Hwang and Yoon, 1981) for solving MCDM problem. It bases upon the concept that the chosen alternative should have the shortest distance from the positive ideal solution (PIS) and the farthest from the negative ideal solution (NIS). In the process of TOPSIS, the performance ratings and the weights of the criteria given as crisp values[5]. Positive ideal solution is a solution that maximizes the benefit criteria and minimizes cost criteria, whereas the negative solution maximizes the cost criteria (Wang and Elhag, 2006). In the classical TOPSIS method, the weights of the criteria and the ratings of alternatives are known precisely and crisp values are used in the evaluation process. However, under many conditions crisp data are inadequate to model real-life decision problems. Therefore, the Fuzzy TOPSIS method is proposed where the weights of criteria and ratings of alternatives are evaluated by entropy crisp numbers to deal with the deficiency in the traditional TOPSIS[2]. The use of fuzzy set theory (Zadeh, 1965) allows the decision-makers to incorporate unquantifiable information, incomplete information; nonobtainable information and partially ignorant facts into decision model (Kulak, Durmusoglu and kahraman, 2005). As a result, fuzzy TOPSIS and its extensions are developed to solve ranking and justification problems. This study uses triangular fuzzy number for fuzzy TOPSIS. The reason for using a triangular fuzzy number is that it is intuitively easy for the decision-makers to numbers has proven to be an effective way for formulating decision problems where the information available is subjective and imprecise. In practical applications, the triangular form of the membership function is used most often for fuzzy numbers (Xu \& Chen, 2007)[8]. In this paper, the concept of TOPSIS is further extended to develop a methodology for solving mutli-person multi-criteria decision making problems in fuzzy environment.

\section{PRELIMINARIES}

The concept of triangular fuzzy number and some operational laws of triangular fuzzy numbers as follows:

\subsection{Definition [10]}

Let $X$ be a nonempty set. A fuzzy set $\tilde{A}$ of $X$ is defined as $\tilde{A}=\left\{\left\langle x, \mu_{\tilde{A}}(x)\right\rangle / x \in X\right\}$ where $\mu_{\tilde{A}}(x)$ is called the membership function which maps each element of $X$ to a value between 0 and 1 .

\subsection{Definition [5]}

A fuzzy set $\tilde{A}$ of the universe of discourse $X$ is called a normal fuzzy set implying that $\exists x \in X, \mu_{\widetilde{A}}(x)=1$.

\subsection{Definition [5]}

A fuzzy set $\tilde{A}$ of the universe of discourse if and only if for all $x_{1}, x_{2}$ in $X, \mu_{\tilde{A}}\left(\lambda x_{1}+(1-\lambda) x_{2}\right) \geq \operatorname{Min}\left(\mu_{\tilde{A}}\left(x_{1}\right), \mu_{\tilde{A}}\left(x_{2}\right)\right)$, where $\lambda \in[0,1]$.

\subsection{Definition [10]}

A fuzzy number is a generalization of a regular real number and which does not refer to a single value but rather to a connected a set of possible values, where each possible values has its weight between 0 and 1 . This weight is called the membership function.

A fuzzy number $\tilde{A}$ is a convex normalized fuzzy set on the real line $R$ such that:

(i)There exist at least one $x \in R$ with $\mu_{\widetilde{A}}(x)=1$.

(ii) $\mu_{\widetilde{A}}(x)$ is piecewise continuous.

\subsection{Definition [8]}

A triangular fuzzy number $\tilde{A}$ can be defined by a trip let $\left(a_{1}, a_{2}, a_{3}\right)$ shown in Fig.1. The membership function $\mu_{\tilde{A}}$ is defined 
$\mu_{\tilde{A}}(x)= \begin{cases}0 & x<a_{1} \\ \frac{x-a_{1}}{a_{2}-a_{1}} & a_{1}<x<a_{2} \\ \frac{x-a_{3}}{a_{2}-a_{3}} & a_{2}<x<a_{3} \\ 0 & x<a_{3}\end{cases}$

$\mu_{\overline{\mathrm{a}}}(x)$

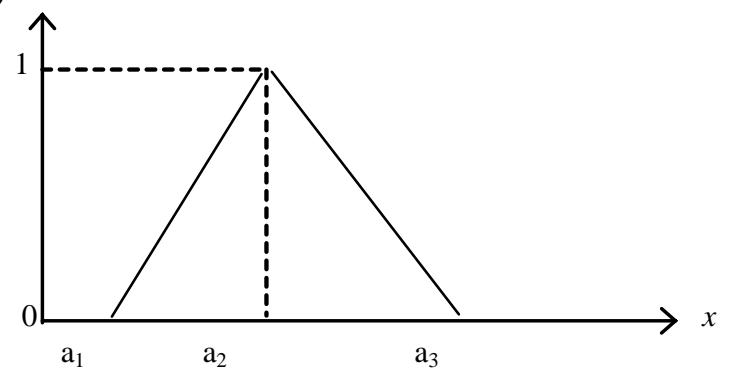

Fig1. A Triangular Fuzzy Number $\tilde{A}$

\subsection{Definition [8]}

Let $\tilde{a}$ and $\tilde{b}$ be two triangular fuzzy numbers parameterized by the triplet $\left(a_{1}, a_{2}, a_{3}\right)$ and $\left(b_{1}, b_{2}, b_{3}\right)$ respectively, then the operational laws of these two triangular fuzzy numbers are as follows:

$$
\begin{aligned}
& \tilde{a}(+) \tilde{b}=\left(a_{1}, a_{2}, a_{3}\right)(+)\left(b_{1}, b_{2}, b_{3}\right)=\left(a_{1}+b_{1}, a_{2}+b_{2}, a_{3}+b_{3}\right) \\
& \tilde{a}(-) \tilde{b}=\left(a_{1}, a_{2}, a_{3}\right)(-)\left(b_{1}, b_{2}, b_{3}\right)=\left(a_{1}-b_{3}, a_{2}-b_{2}, a_{3}-b_{1}\right) \\
& \tilde{a}(\times) \tilde{b}=\left(a_{1}, a_{2}, a_{3}\right)(\times)\left(b_{1}, b_{2}, b_{3}\right)=\left(a_{1} \times b_{1}, a_{2} \times b_{2}, a_{3} \times b_{3}\right) \\
& \tilde{a}(/) \tilde{b}=\left(a_{1}, a_{2}, a_{3}\right)(/)\left(b_{1}, b_{2}, b_{3}\right)=\left(a_{1} / b_{3}, a_{2} / b_{2}, a_{3} / b_{1}\right) \\
& \tilde{a}=\left(k a_{1}, k a_{2}, k a_{3}\right)
\end{aligned}
$$

\subsection{Definition [8]}

Let $\tilde{a}=\left(a_{1}, a_{2}, a_{3}\right)$ and $\tilde{b}=\left(b_{1}, b_{2}, b_{3}\right)$ be two triangular fuzzy numbers, then the vertex method is defined to calculate the distance between them,

$$
d(\tilde{a}, \tilde{b})=\sqrt{\frac{1}{3}\left[\left(a_{1}-b_{1}\right)^{2}+\left(a_{2}-b_{2}\right)^{2}+\left(a_{3}-b_{3}\right)^{2}\right]}
$$

\subsection{Definition [2]}

If $\tilde{A}=\left(a_{1}, a_{2}, a_{3}\right), \widetilde{B}=\left(b_{1}, b_{2}, b_{3}\right)$ are two triangular fuzzy numbers, then the distance of $\widetilde{A}$ from $\widetilde{B}$ is achieved by following relation:

$$
S(\widetilde{B}, \widetilde{A})=\frac{\left(b_{1}+2 b_{2}+b_{3}\right)-\left(a_{1}+2 a_{2}+a_{3}\right)}{4}
$$

It is clear the distance of the triangular fuzzy number $\tilde{A}$ the crisp number 0 equals following value:

$$
S(\tilde{A}, 0)=\frac{\left(a_{1}+2 a_{2}+a_{3}\right)}{4}
$$

\section{PROPOSED METHOD}

The steps of the proposed fuzzy TOPSIS method are following:

Step 1:A decision matrix for ranking is established and a MCDM problem can be concisely expressed in matrix format as:

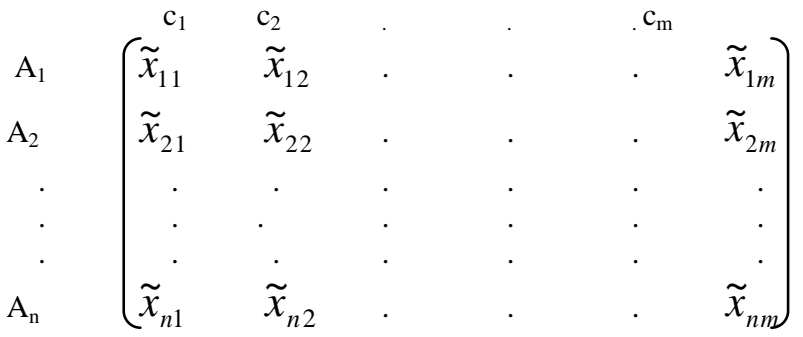

Where $A_{1}, A_{2}, A_{3}, \ldots, A_{n}$ are possible alternatives among which decision makers have to choose, $C_{1}, C_{2}, C_{3}, \ldots, C_{m}$ are criteria with which alternative performance are measured, $\tilde{x}_{i j}=\left(x_{i j}^{a}, x_{i j}^{b}, x_{i j}^{c}\right)$ is the fuzzy rating of alternative, $A_{i}$ with respect to criterion $C_{j}$.

Step2:The normalized decision matrix is calculated and the value of $\tilde{n}_{i j}=\left(n_{i j}^{a}, n_{i j}^{b}, n_{i j}^{c}\right)$ where

$\tilde{n}_{i j}=\frac{\tilde{x}_{i j}}{\sqrt{\sum_{i=1}^{n}\left(s\left(\tilde{x}_{i j}, 0\right)\right)^{2}}}, j=1,2,3, \ldots, n$

where $s\left(\tilde{x}_{i j}, 0\right)=\frac{x_{i j}^{a}+2 x_{i j}^{b}+x_{i j}^{c}}{4}$

Step3: The weighted normalized decision matrix is calculated and the output entropy $e_{j}$ of the $j^{\text {th }}$ factor becomes

$e_{j}=-k \sum_{i=1}^{m} p_{i j} \ln p_{i j},(k=1 / \ln m, 1 \leq j \leq n)$

Variation coefficient of the $j^{\text {th }}$ factor $g_{j}$ can be defined by the following equation:

$$
d_{j}=1-e_{j},(1 \leq j \leq n)
$$

Calculate the weight of the entropy $w_{j}$ :

$$
w_{j}=g_{j} / \sum_{i=1}^{m} g_{j}, \quad(1 \leq j \leq n)
$$

Step4: The weighted normalized value $\tilde{V}_{i j}=\left(v_{i j}^{a}, v_{i j}^{b}, v_{i j}^{c}\right)$ is calculated, considering the different importance values of each criterion and, the weighted normalized fuzzy-decision matrix is constructed as, if $W$ is a crisp value: $\widetilde{V}=\left[\widetilde{V}_{i j}\right]_{n \times m}, i=1,2,3, \ldots, n, j=1,2,3, \ldots, m$, where $\tilde{V}_{i j}=\tilde{x}_{i j} \times W_{i}, W_{j}$ is the weight if the $i^{t h}$ criterion, and $\sum_{j=1}^{n} W_{j}=1$. 
A set of performance ratings of $A_{i}=(i=1,2,3, \ldots, n)$ with respect to criteria $C_{j}=(j=1,2,3, \ldots, m)$ called $\tilde{x}=\left(\tilde{x}_{i j}, i=1,2, \ldots, n, j=1,2, \ldots, m\right) . \quad$ A $\quad$ set $\quad$ of importance weights of each criterion $W_{i}=(i=1,2,3, \ldots, n)$.

Step 5: The positive ideal solutions and the negative ideal solutions are determined respectively:

$$
\begin{aligned}
& A^{+}=\left\{\tilde{v}_{1}^{+}, \tilde{v}_{2}^{+}, \ldots . ., \tilde{v}_{n}^{+}\right\} \\
& A^{-}=\left\{\tilde{v}_{1}^{-}, \tilde{v}_{2}^{-}, \ldots . ., \tilde{v}_{n}^{-}\right\}
\end{aligned}
$$

Step 6:The separation measures using the $\mathrm{n}$-dimensional Euclidean distance is calculated as

$$
S(\widetilde{B}, \widetilde{A})=\frac{\left(b_{1}+2 b_{2}+b_{3}\right)-\left(a_{1}+2 a_{2}+a_{3}\right)}{4}
$$

Step 7: The relative closeness to the ideal solution is calculated and the relative closeness of the alternative $A_{i}$ with respect to $A^{+}$is defined as:

$$
c l_{i}^{+}=\frac{d_{i}^{+}}{d_{i}^{+}+d_{i}^{-}}, \quad i=1,2, \ldots ., n
$$

Step 8: The preference order is ranked and the highest value is the better alternative.

\section{NUMERICAL EXAMPLE}

Table 1 describes the details of the 5 different crops in fuzzy numbers collected from Tamil Nadu Agricultural University. The alternatives $A_{1}, A_{2}, A_{3}, A_{4}, A_{5}$ are rice, groundnut, maize, ragi, blackgram according to the criteria $C_{1}, C_{2}, C_{3}, C_{4}, C_{5}$ are the duration, water requirement, Productivity, Quantity of water required, Water Use Efficiency respectively.

Step 1:

Table 1. Collected data

\begin{tabular}{|l|l|l|l|l|l|}
\hline Criteria & $\begin{array}{l}\text { Durat } \\
\text { ion } \\
\text { (Days) }\end{array}$ & $\begin{array}{l}\text { Water } \\
\text { Require } \\
\text { ives } \\
\text { ment } \\
(\mathbf{m m})\end{array}$ & $\begin{array}{l}\text { Producti } \\
\text { vity } \\
\mathbf{( k g ~ m}^{-3} \mathbf{)}\end{array}$ & $\begin{array}{l}\text { Quant } \\
\text { ity of } \\
\text { water } \\
\text { requir } \\
\text { ed } \\
\left.\mathbf{( m}^{3} \mathbf{k g}^{-1}\right)\end{array}$ & $\begin{array}{l}\text { Water } \\
\text { Use } \\
\text { Efficie } \\
\text { ncy } \\
\mathbf{k g ~ h a}^{-1} \\
\mathbf{m m}^{-1} \mathbf{)}\end{array}$ \\
\hline Rice & $(90$, & $(1240$, & $(0.31$, & $(2.34$, & $(4.10$, \\
& 100, & 1250, & 0.41, & 2.44, & 4.20, \\
& $110)$ & $1260)$ & $0.51)$ & $2.55)$ & $4.30)$ \\
\hline Groundn & $(95$, & $(500$, & $(0.21$, & $(4.06$, & $(2.00$, \\
ut & 105, & 510, & 0.31, & 4.16, & 2.10, \\
& $115)$ & $520)$ & $0.41)$ & $4.26)$ & $2.20)$ \\
\hline Maize & $(90$, & $(490$, & $(0.73$, & $(0.98$, & $(9.10$, \\
& 100, & 500, & 0.83, & 1.08, & $9.20,9$. \\
& $110)$ & $510)$ & $0.93)$ & $1.18)$ & $30)$ \\
\hline Ragi & $(85$, & $(300$, & $(0.68$, & $(1.03$, & $(8.60$, \\
& 95, & 310, & 0.78, & 1.13, & 8.70, \\
& $105)$ & $320)$ & $0.88)$ & $1.23)$ & $8.80)$ \\
\hline Blackgra & $(65$, & $(280$, & $(0.15$, & $(3.90$, & $(2.50$, \\
& 75, & 290, & 0.25, & 4.00, & 2.60, \\
& $85)$ & $300)$ & $0.35)$ & $4.10)$ & $2.70)$ \\
\hline
\end{tabular}

Step2:

Table 2: Fuzzy Decision Matrix

\begin{tabular}{|l|l|l|l|l|l|}
\hline $\mathbf{C}$ & $\mathbf{C}_{\boldsymbol{1}}$ & $\mathbf{C}_{\mathbf{2}}$ & $\mathbf{C}_{\mathbf{3}}$ & $\mathbf{C}_{\mathbf{4}}$ & $\mathbf{C}_{\mathbf{5}}$ \\
\hline \multirow{4}{*}{$\mathrm{A}_{1}$} & $(0.4211$, & $(0.8262$, & $(0.2433$, & $(0.3623$, & $(0.2981$, \\
& 0.4679, & 0.8328, & 0.3217, & 0.3778, & 0.3054, \\
& $0.5147)$ & $0.8395)$ & $0.4002)$ & $0.3948)$ & $0.3127)$ \\
\hline \multirow{4}{*}{$\mathrm{A}_{2}$} & $(0.4445$, & $(0.3331$, & $(0.1648$, & $(0.6286$, & $(0.1454$, \\
& 0.4913, & 0.3398, & 0.2433, & 0.6441, & 0.1527, \\
& $0.5381)$ & $0.3465)$ & $0.3217)$ & $0.6596)$ & $0.1600)$ \\
\hline \multirow{4}{*}{$\mathrm{A}_{3}$} & $(0.4211$, & $(0.3265$, & $(0.7690$, & $(0.1517$, & $(0.6617$, \\
& 0.4679, & 0.3331, & 0.8475, & 0.1672, & 0.6690, \\
& $0.5147)$ & $0.3398)$ & $0.9259)$ & $0.1827)$ & $0.6762)$ \\
\hline \multirow{4}{*}{$\mathrm{A}_{4}$} & $(0.3977$, & $(0.1999$, & $(0.5336$, & $(0.1595$, & $(0.6253$, \\
& 0.4100, & 0.2065, & 0.6121, & 0.1750, & 0.6326, \\
& $0.4913)$ & $0.2132)$ & $0.6910)$ & $0.1904)$ & $0.6399)$ \\
\hline \multirow{4}{*}{$\mathrm{A}_{5}$} & $(0.3041$, & $(0.1866$, & $(0.1177$, & $(0.6038$, & $(0.1818$, \\
& 0.3509, & 0.1932, & 0.1962, & 0.6193, & 0.1891, \\
& $0.3977)$ & $0.1999)$ & $0.2746)$ & $0.6348)$ & $0.1963)$ \\
\hline
\end{tabular}

Step3: For the weight using entropy analysis, the procedure

\begin{tabular}{|c|c|c|c|c|c|}
\hline Criteria & & & & & \\
\hline Alternatives & $\mathrm{C}_{1}$ & $\mathbf{C}_{2}$ & $\mathbf{C}_{3}$ & $\mathrm{C}_{4}$ & $\mathrm{C}_{5}$ \\
\hline $\mathrm{A}_{1}$ & 0.2315 & 0.4366 & 0.1529 & 0.1890 & 0.1529 \\
\hline $\mathbf{A}_{2}$ & 0.2211 & 0.1796 & 0.0784 & 0.3299 & 0.0784 \\
\hline $\mathbf{A}_{3}$ & 0.2105 & 0.1761 & 0.3470 & 0.0836 & 0.3470 \\
\hline $\mathbf{A}_{4}$ & 0.2000 & 0.1092 & 0.3284 & 0.0875 & 0.3284 \\
\hline $\mathbf{A}_{5}$ & 0.1368 & 0.0986 & 0.0932 & 0.3098 & 0.0933 \\
\hline
\end{tabular}
is as follows, the fuzzy decision matrix shown in Table2.

$$
\begin{aligned}
& P_{i j}=\frac{x_{i j}}{\sum_{i=1}^{m} x_{i j}}(1 \leq i \leq m, 1 \leq j \leq n) \\
& \sum_{i=1}^{5} x_{i 1}=475 \\
& P_{11}=\frac{110}{475}=0.2315 \quad P_{21}=0.2211 P_{31}=0.2105
\end{aligned}
$$

Table 3. Entropy Normalization Matrix

To find the value of $P_{i j} \ln \left(P_{i j}\right)$

$P_{11} \ln \left(P_{11}\right)=0.2315 \ln (0.2315)=-0.3387$ 
Table 4. Weight Calculations Matrix

\begin{tabular}{|l|l|l|l|l|l|}
\hline \multicolumn{1}{|l|}{ Criteria } & \multirow{2}{*}{$\begin{array}{l}\mathrm{C}_{1} \\
\text { Alternati } \\
\text { ves }\end{array}$} & $\mathrm{C}_{2}$ & $\mathrm{C}_{3}$ & $\mathrm{C}_{4}$ & $\mathrm{C}_{5}$ \\
\hline $\mathrm{A}_{1}$ & -0.3387 & -0.3618 & -0.2871 & -0.3149 & -0.2870 \\
\hline $\mathrm{A}_{2}$ & -0.3337 & -0.3084 & -0.1996 & -0.3658 & -0.1995 \\
\hline $\mathrm{A}_{3}$ & -0.3280 & -0.3058 & -0.3673 & -0.2075 & -0.3673 \\
\hline $\mathrm{A}_{4}$ & -0.3219 & -0.2418 & -0.3657 & -0.2132 & -0.3657 \\
\hline $\mathrm{A}_{5}$ & -0.2721 & -0.2284 & -0.2212 & -0.3630 & -0.2212 \\
\hline
\end{tabular}

$e_{j}=-k \sum_{i=1}^{m} p_{i j} \ln p_{i j},(k=1 / \ln m, 1 \leq j \leq n)$

$k=\frac{1}{\ln (5)}=0.6212$

$e_{1}=-0.6212(-1.5944)=0.9903$

$e_{2}=-0.6212(-1.4462)=0.8984$

$d_{1}=1-e_{1}=1-0.9903=0.0097$

$d_{2}=1-0.8984=0.1016$

$w_{j}=\frac{1-e_{j}}{n-\sum_{j=1}^{n} e_{j}}$

$n-\sum_{j=1}^{5} e_{j}=5-4.5884=0.4116\left\lfloor\therefore 1-e_{j}=d_{j}\right\rfloor$

$w_{1}=\frac{d_{1}}{0.4116}=0.0219$

$w_{2}=\frac{0.1016}{0.4116}=0.2467$

Table 5: Entropy Weight calculations

\begin{tabular}{|l|l|l|l|l|l|}
\hline Criteria & $\mathrm{C}_{1}$ & $\mathrm{C}_{2}$ & $\mathrm{C}_{3}$ & $\mathrm{C}_{4}$ & $\mathrm{C}_{5}$ \\
\hline $\mathbf{E}_{\mathbf{j}}$ & 0.9903 & 0.8984 & 0.8951 & 0.9097 & 0.8949 \\
\hline $\mathbf{d}_{\mathbf{j}}$ & 0.0097 & 0.1016 & 0.1049 & 0.0903 & 0.1051 \\
\hline $\mathbf{w}_{\mathbf{j}}$ & 0.0219 & 0.2467 & 0.2549 & 0.2194 & 0.2552 \\
\hline
\end{tabular}

Step4: To find the value of $\tilde{V}_{i j}=W_{j} \times \tilde{n}_{i j}, i=1,2, \ldots . ., n, j=1,2, \ldots ., m$
Table 6. Fuzzy Normalized Matrix

\begin{tabular}{|c|c|c|c|c|c|}
\hline $\mathrm{C}^{\mathrm{C}}$ & $\mathbf{C}_{1}$ & $\mathbf{C}_{2}$ & $\mathbf{C}_{3}$ & $\mathrm{C}_{4}$ & $\mathrm{C}_{5}$ \\
\hline $\mathbf{A}_{1}$ & $\begin{array}{l}(0.0092 \\
0.0103 \\
0.0113)\end{array}$ & $\begin{array}{l}(0.2038, \\
0.2055, \\
0.2071)\end{array}$ & $\begin{array}{l}(0.0620, \\
0.0820, \\
0.1020)\end{array}$ & $\begin{array}{l}(0.0716, \\
0.0829, \\
0.0866)\end{array}$ & $\begin{array}{l}(0.0761, \\
0.0779, \\
0.0798)\end{array}$ \\
\hline $\mathbf{A}_{2}$ & $\begin{array}{l}(0.0097 \\
0.0108 \\
0.0118)\end{array}$ & $\begin{array}{l}(0.0822, \\
0.0838, \\
0.0855)\end{array}$ & $\begin{array}{l}(0.0420, \\
0.0620, \\
0.0820)\end{array}$ & $\begin{array}{l}(0.1379, \\
0.1413, \\
0.1447)\end{array}$ & $\begin{array}{l}(0.0371, \\
0.0390, \\
0.0408)\end{array}$ \\
\hline $\mathbf{A}_{3}$ & $\begin{array}{l}(0.0092 \\
0.0103 \\
0.0113)\end{array}$ & $\begin{array}{l}(0.0806, \\
0.0822, \\
0.0838)\end{array}$ & $\begin{array}{l}(0.1960, \\
0.2160, \\
0.2360)\end{array}$ & $\begin{array}{l}(0.0333, \\
0.0367, \\
0.0401)\end{array}$ & $\begin{array}{l}(0.1689, \\
0.1707, \\
0.1726)\end{array}$ \\
\hline $\mathbf{A}_{4}$ & $\begin{array}{l}(0.0087 \\
0.0090 \\
0.0108)\end{array}$ & $\begin{array}{l}(0.0493, \\
0.0509, \\
0.0526)\end{array}$ & $\begin{array}{l}(0.1360, \\
0.1560, \\
0.1761)\end{array}$ & $\begin{array}{l}(0.0350, \\
0.0384, \\
0.0418)\end{array}$ & $\begin{array}{l}(0.1596, \\
0.1614, \\
0.1633)\end{array}$ \\
\hline $\mathbf{A}_{5}$ & $\begin{array}{l}(0.0067, \\
0.0077 \\
0.0087)\end{array}$ & $\begin{array}{l}(0.0460, \\
0.0477, \\
0.0493)\end{array}$ & $\begin{array}{l}(0.0300, \\
0.0491, \\
0.0700)\end{array}$ & $\begin{array}{l}(0.1325, \\
0.1359, \\
0.1393)\end{array}$ & $\begin{array}{l}(0.0464, \\
0.0483, \\
0.0501)\end{array}$ \\
\hline
\end{tabular}

Step 5:To find the negative and positive ideal solution:

$S(\widetilde{A}, 0)=\frac{\left(a_{1}+2 a_{2}+a_{3}\right)}{4}$

$S((0.0092,0.0103,0.0113), 0)=\frac{0.0092+0.0206+0.0113}{4}$

$$
=0.0103
$$

$S((0.0097,0.0108,0.0118), 0)=\frac{0.0097+0.0216+0.0118}{4}$ $=0.01077$

$S((0.0087,0.0090,0.0108), 0)=\frac{0.0087+0.018+0.0108}{4}$ $=0.0094$

$S((0.0067,0.0077,0.0087), 0)=\frac{0.0067+0.0154+0.0087}{4}$ $=0.0077$

$A^{+}=\left\{\begin{array}{l}(0.0097,0.0108,0.0118),(0.2038,0.2055,0.2071), \\ (0.1960,0.2160,0.2360),(0.1379,0.1413,0.1447), \\ (0.1689,0.1707,0.1726)\end{array}\right\}$
$A^{-}=\left\{\begin{array}{l}(0.0067,0.0077,0.0087),(0.0460,0.0477,0.0493), \\ (0.0300,0.0491,0.0700),(0.0333,0.0367,0.0401), \\ (0.0371,0.0390,0.0408)\end{array}\right\}$

Step6:

$S(\tilde{B}, \tilde{A})=\frac{\left(b_{1}+2 b_{2}+b_{3}\right)-\left(a_{1}+2 a_{2}+a_{3}\right)}{4}$

$d_{1}^{+}=\sqrt{0.00000025+0+0.017956+0.00363604+0.008612}$

$=0.173794$ 


$$
c l_{1}^{+}=\frac{d_{1}^{+}}{d_{1}^{+}+d_{1}^{-}}=\frac{0.173794}{0.173794+0.172102}=0.5025
$$

Table 7. Ranking

\begin{tabular}{|l|l|l|l|l|}
\hline Alternatives & $d_{i}^{+}$ & $d_{i}^{-}$ & $c_{i}^{+}$ & Ranking \\
\hline Rice & 0.173794 & 0.172102 & 0.5025 & 4 \\
\hline Groundnut & 0.236375 & 0.111414 & 0.6797 & 2 \\
\hline Maize & 0.161673 & 0.215088 & 0.4291 & 5 \\
\hline Ragi & 0.195343 & 0.162321 & 0.5462 & 3 \\
\hline Blackgram & 0.260077 & 0.099634 & 0.7230 & 1 \\
\hline
\end{tabular}

As shown in above table, the final ranking is based on the highest value of $\mathrm{Cl}_{i}^{+}$. We get the highest value, Blackgram is 0.7230 .

\section{CONCLUSION}

Decision-makers in most cases reach a situation of uncertainty and vagueness from subjective perceptions and experiences in the process. By using fuzzy TOPSIS, this can be effectively represented and reach to a more effective outcome. In this paper a new method has been presented to expand TOPSIS decision making model to fuzzy TOPSIS with triangular fuzzy numbers. Fuzzy TOPSIS method is used to obtain final ranking. Similar calculations are done for the other alternatives and the results of fuzzy TOPSIS analyses are summarized. So, this is a better and more accurate outputs in comparison with previous method. Based on $\mathrm{Cl}_{i}^{+}$values, the ranking in descending order is $A_{5}, A_{2}, A_{4}, A_{1}$ and $A_{3}$. In the proposed method, obtained the highest value $A_{5}$ which is blackgram have fulfilled the criteria's duration, water requirement, Productivity, Quantity of water required, Water Use Efficiency.

\section{REFERENCES}

[1] Atanassov.K.T., Intuitionistic fuzzy sets, Fuzzy sets and systems, Vol.20, No.1 (1986) 87-96.

[2] Ali mohammad, Abolfazlmohammadi, Hossain aryaeefar, Introduction a new method to expand TOPSIS decision making model to Fuzzy TOPSIS, The Journal of mathematics and Computer Science Vol.2. No.1 (2011) 150-159.

[3] Chang, Y.H., \&Yeh, C.H. (2002), A survey analysis of service quality for domestic airlines, European Journal of Operational Research, 139, 166-177.

[4] Chen, T.Y., \&Tsao, C. Y. (2007), The interval-valued fuzzy TOPSIS methods and experimental analysis, Fuzzy Sets and Systems.

[5] Chen-Tung Chen, Extension of the TOPSIS for group decision-making under Fuzzy environment, Elsevier, Fuzzy Sets and Systems 114 (2000) 1-9.

[6] Irajalavi and Hamid Alinejad-Rokny, Comparison of Fuzzy AHP and Fuzzy TOPSIS Methods for plant species selection (Case study: Reclamation Plan of Sungun Copper Mine; Iran), Australian Journal of Basic and Applied Sciences, 5 (12), (2011) 1104-1113.

[7] Hwang.C.L., \&Yoon.K, Multiple Attributes Decision Making Methods and Applications, Springer, Berlin Heidelberg, 1981.

[8] MortezaPakdinAmiri, Project selection for oil-fields development by using the AHP and fuzzy TOPSIS methods, Elsevier, Expert Systems with Applications 37 (2010) 6218-6224.

[9] SahayaSudha.A, Rachel InbaJeba.J, Selection of Planting of Crops by Rotation Using TOPSIS, Journal of Global Research in Mathematical Archives, Vol.2, No.6, (2014) 15-20.

[10] Thamraiselvi.A and Santhi.R, On Intuitionistic Fuzzy Transportation Problems Using Hexagonal Intuitionistic Fuzzy Numbers, International Journal of Fuzzy Logic Systems (IJFLS) Vol.5, No.1, January 2015.

[11] Zadeh.L.A., Fuzzy Sets, Inform and control 8 (1965) 338-353. 